\title{
Exponential stability for differential equations with random impulses at random times
}

\author{
Ravi Agarwal ${ }^{1 *}$, Snezhana Hristova ${ }^{2}$ and Donal O'Regan ${ }^{3}$
}

${ }^{\text {"Correspondence: }}$

agarwal@tamuk.edu

1 Department of Mathematics, Texas A\&M University-Kingsville, Kingsville, 78363, USA

Full list of author information is available at the end of the article

\begin{abstract}
Impulsive differential equations with impulses occurring at random times arise in the modeling of real world phenomena in which the state of the investigated process changes instantaneously at uncertain moments. The investigation of these differential equations uses ideas in the qualitative theory of differential equations and probability theory. In this paper differential equations with randomly occurring impulses are considered and the $p$-moment exponential stability of the solutions is studied.
\end{abstract}

MSC: 34A37; 34E05

Keywords: impulsive differential equations; random moments of impulses; p-moment exponential stability

\section{Introduction}

Impulsive differential equations are studied extensively in the literature. Many authors consider impulsive differential equations with determined impulsive moments (see, for example, the monographs [1-4] and the references cited therein). However, in some real world phenomena the investigated process changes instantaneously at uncertain moments. When modeling such processes, it is necessary to use random variables in jump conditions and impulsive differential equations with random impulses occurring at random moments. The presence of randomness in the jump condition changes the behavior of solutions of differential equations significantly. In the case of impulses occurring at random moments, the solution is a stochastic process.

In the literature a number of results have been obtained for stochastic differential equations with jumps [5-7]. Also, some results on the qualitative properties of equations with random impulses have been obtained [8-10]. In the monograph [11], impulsive differential equations with fixed impulses and random amplitude of jumps were studied.

In this paper we study nonlinear differential equations subject to random impulses occurring at random moments. Randomness is introduced both through the time between impulses, which is distributed exponentially, and through the amount of impulses. The $p$-moment exponential stability of the solution is studied by employing appropriate generalized Lyapunov's functions. In the literature many authors study the stability of impulsive systems with deterministic moments of impulses (see $[1,2,4]$ and the references cited therein), the exponential stability of impulsive delay differential equations with deterministic moments of impulses [12-15] and the $p$-moment stability of stochastic differential equations with or without impulses [6, 7, 16,17]. The behavior of solutions of stochastic equations is totally different from the behavior of ordinary differential equations, so the

(อ2013 Agarwal et al.: licensee Springer. This is an Open Access article distributed under the terms of the Creative Commons Attribution License (http://creativecommons.org/licenses/by/2.0), which permits unrestricted use, distribution, and reproduction in any medium, provided the original work is properly cited. 
study of stability properties of differential equations with impulses occurring at random moments is important.

\section{Preliminary notes and results}

Let the probability space $(\Omega, \mathcal{F}, P)$ be given. Let $\left\{\tau_{k}\right\}_{k=1}^{\infty}$ be a sequence of independent exponentially distributed random variables with a parameter $\lambda>0$ that are defined on the sample space $\Omega$. We will call the random variables $\tau_{k}$ waiting times since they will define the time between two consecutive impulses of the considered impulsive differential equation.

Define the sequence of random variables $\left\{\xi_{k}\right\}_{k=0}^{\infty}$ such that $\xi_{0}=T_{0}$ and $\xi_{k}=T_{0}+\sum_{i=1}^{k} \tau_{i}$, $k=1,2, \ldots$, where $T_{0} \geq 0$ is a fixed point.

We note that $\left\{\xi_{k}\right\}_{k=0}^{\infty}$ is an increasing sequence of random variables.

Assume $\sum_{k=1}^{\infty} \tau_{k}=\infty$ with probability 1 .

Let $t \geq T_{0}$ be a fixed point. Consider the events

$$
S_{k}(t)=\left\{\omega \in \Omega: \xi_{k}(\omega)<t<\xi_{k+1}(\omega)\right\}, \quad k=1,2, \ldots
$$

Let the points $t_{k}$ be arbitrary values of the random variables $\tau_{k}, k=1,2, \ldots$, correspondingly. Define the increasing sequence of points $T_{k}=\sum_{i=0}^{k} t_{i}, k=1,2,3, \ldots$, that are values of the random variables $\xi_{k}$. Consider the initial value problem for the scalar impulsive differential equation with fixed points of impulses

$$
\begin{aligned}
& x^{\prime}=f(t, x(t)) \quad \text { for } t \geq T_{0}, t \neq T_{k}, \\
& x\left(T_{k}+0\right)=I_{k}\left(t_{k}, x\left(T_{k}-0\right)\right) \text { for } k=1,2, \ldots, \\
& x\left(T_{0}\right)=x_{0},
\end{aligned}
$$

where $x \in \mathbb{R}^{n}, f:[0, \infty) \times \mathbb{R}^{n} \rightarrow \mathbb{R}^{n}, I_{k}:[0, \infty) \times \mathbb{R}^{n} \rightarrow \mathbb{R}^{n}$ and $x_{0} \in \mathbb{R}^{n}$.

The solution of the impulsive differential equation with fixed moments of impulses (1) depends not only on the initial condition $\left(T_{0}, x_{0}\right)$ but on the moments of impulses $T_{k}$, $k=1,2, \ldots$, i.e., the solution depends on the initially chosen arbitrary values $t_{k}$ of the random variables $\tau_{k}, k=1,2, \ldots$. We denote the solution of initial value problem (1) by $x\left(t ; T_{0}, x_{0},\left\{t_{k}\right\}\right)$. We will assume that $x\left(T_{k} ; T_{0}, x_{0},\left\{t_{k}\right\}\right)=\lim _{t \rightarrow T_{k}-0} x\left(t ; T_{0}, x_{0},\left\{t_{k}\right\}\right)$.

Remark 1 We note that the length of the interval $\left(T_{k}, T_{k+1}\right)$ of the continuity of the solutions of the initial value problem for impulsive differential equation with fixed moments of impulses (1) is equal to $t_{k}$, that is, a value of the random variable $\tau_{k}$, called the waiting time.

The set of all solutions $x\left(t ; T_{0}, x_{0},\left\{t_{k}\right\}\right)$ of the initial value problem for the impulsive differential equation (1) for any values $t_{k}$ of the random variables $\tau_{k}, k=1,2, \ldots$, generates a stochastic process with state space $\mathbb{R}^{n}$. We denote it by $x\left(t ; T_{0}, x_{0},\left\{\tau_{k}\right\}\right)$ and we will say that it is a solution of the following initial value problem for impulsive differential equations with random moments of impulses (RIDE):

$$
\begin{aligned}
& x^{\prime}=f(t, x(t)) \quad \text { for } t \geq T_{0}, \xi_{k}<t<\xi_{k+1}, \\
& x\left(\xi_{k}+0\right)=I_{k}\left(\tau_{k}, x\left(\xi_{k}-0\right)\right) \text { for } k=1,2, \ldots, \\
& x\left(T_{0}\right)=x_{0} .
\end{aligned}
$$


For any values $t_{k}$ of the random variables $\tau_{k}$, the solution $x\left(t ; T_{0}, x_{0},\left\{t_{k}\right\}\right)$ of the initial value problem for the impulsive equation with fixed points of impulses (1) will be called $a$ sample path solution of RIDE (2).

Define the stochastic processes $\Delta_{k}(t), k=1,2, \ldots$, by the equality

$$
\Delta_{k}(t)= \begin{cases}1 & \text { for } \omega \in S_{k}(t), \\ 0 & \text { for } \omega \notin S_{k}(t) .\end{cases}
$$

We note that for any fixed point $t$ and any element $\omega \in \Omega$, there exists a natural number $k$ such that $\omega \in S_{k}(t)$ and $\omega \notin S_{j}(t)$ for $j \neq k$, or for any fixed point $t$, there exists a natural number $k$ such that $\Delta_{k}(t)=1$ and $\Delta_{j}(t)=0$ for $j \neq k$.

We will prove the following result for the stochastic processes $\Delta_{k}(t)$.

Lemma 2.1 Let $\left\{\tau_{k}\right\}_{1}^{\infty}$ be independent exponentially distributed random variables (IED) with a parameter $\lambda$ and $\xi_{k}=T_{0}+\sum_{i=1}^{k} \tau_{i}$.

Then

$$
E\left(\Delta_{k}(t)\right)=\frac{\lambda^{k}\left(t-T_{0}\right)^{k}}{k !} e^{-\lambda\left(t-T_{0}\right)} \quad \text { for } t \geq T_{0} \text { and } k=1,2, \ldots
$$

Proof Using the distribution of random variables $\tau_{k}$, the definition of the mean of joint distributed exponentially random variables, and the definition of the sequence of random variables $\left\{\xi_{k}\right\}_{1}^{\infty}$, we obtain

$$
\begin{aligned}
E\left(\Delta_{k}(t)\right) & =\lambda^{k+1} \underbrace{\int \cdots \int}_{\sum_{i=1}^{k} \theta_{i} \leq t-T_{0}<\sum_{i=1}^{k+1} \theta_{i}} e^{-\lambda\left(\theta_{1}+\cdots+\theta_{k+1}\right)} d \theta_{1} \cdots d \theta_{k+1} \\
& =\lambda^{k} e^{-\lambda\left(t-T_{0}\right)} \underbrace{\int \cdots \int}_{\sum_{i=1}^{k} \theta_{i} \leq t-T_{0}} d \theta_{1} \cdots d \theta_{k} \quad \text { for } t \geq T_{0} .
\end{aligned}
$$

From (5) and the equality

$$
\underbrace{\int \cdots \int}_{\sum_{i=1}^{k} \theta_{i} \leq t} d \theta_{1} \cdots d \theta_{k}=\frac{t^{k}}{k !},
$$

we have (4).

Corollary 1 The probability that there will be exactly $k$ impulses until the time $t, t \geq T_{0}$, is given by the equality

$$
P\left(S_{k}(t)\right)=\frac{\lambda^{k}\left(t-T_{0}\right)^{k}}{k !} e^{-\lambda\left(t-T_{0}\right)} .
$$

Proof The result follows immediately from the definition of the event $S_{k}(t)$, Lemma 2.1 and the fact that $P\left(S_{k}(t)\right)=E\left(\Delta_{k}(t)\right)$. 
Now we will illustrate some differences between impulsive differential equations with deterministic moments of impulses and differential equations with randomly occurring impulses.

Example 1 (Ordinary differential equation) Consider the IVP

$$
x^{\prime}=0, \quad x(0)=x_{0} \neq 0 .
$$

The solution of $(6) x(t)=x_{0}$ is stable but is not approaching 0 .

Example 2 (Impulsive differential equations with fixed points of impulses) Let the increasing sequence of points $T_{i}, i=1,2, \ldots$, be given and $\lim _{k \rightarrow \infty} T_{k}=\infty$. Consider the initial value problem (IVP) for the impulsive differential equation

$$
\begin{aligned}
& x^{\prime}=0 \quad \text { for } t \geq 0, t \neq T_{k}, \\
& x\left(T_{k}+0\right)=a x\left(T_{k}-0\right) \quad \text { for } k=1,2, \ldots, \\
& x(0)=x_{0} \neq 0 .
\end{aligned}
$$

The solution of IVP (7) is $x(t)=a^{k} x_{0}$ for $t \in\left(T_{k}, T_{k+1}\right]$.

The solution is a piecewise continuous function.

The behavior of $x(t)$ depends significantly on the amplitude of jumps.

If $|a|<1$, then $|x(t)|$ is approaching 0 (different from the corresponding ordinary differential equation considered in Example 1).

Example 3 (Impulsive differential equation with random points of impulses) Let the sequence of independent exponentially distributed random variables $\tau_{i}, i=1,2, \ldots$ (waiting time) be given. Define $\xi_{k}=\sum_{j=1}^{k} \tau_{k}$ (moments of impulses).

Consider the IVP for the impulsive differential equation with random moments of impulses

$$
\begin{aligned}
& x^{\prime}=0 \quad \text { for } t \geq 0, \xi_{k}<t<\xi_{k+1}, \\
& x\left(\xi_{k}+0\right)=a x\left(\xi_{k}-0\right) \quad \text { for } k=1,2, \ldots, \\
& x(0)=x_{0} \neq 0 .
\end{aligned}
$$

Let, for any $k=1,2, \ldots$, the point $t_{k}$ be an arbitrary value of the random variable $\tau_{k}$. Define the increasing sequence of points $T_{k}=\sum_{i=0}^{k} t_{i}, k=1,2,3 \ldots$, that are values of the random variables $\xi_{k}$.

Consider the IVP for the corresponding impulsive differential equation with fixed points of impulses

$$
\begin{aligned}
& x^{\prime}=0 \quad \text { for } t \geq 0, t \neq T_{k}, \\
& x\left(T_{k}+0\right)=a x\left(T_{k}-0\right) \quad \text { for } k=1,2, \ldots, \\
& x(0)=x_{0} .
\end{aligned}
$$

The solution of (9) is $x(t)=a^{k} x_{0}$ for $T_{k}<t \leq T_{k+1}$. 
It depends not only on $x_{0}$ but on the moments of impulses $T_{k}$, i.e., on the initially chosen arbitrary values $t_{k}$ of the random variables $\tau_{k}, k=1,2, \ldots$.

The set of all solutions of IVP (9) for any values $t_{k}$ of the random variables $\tau_{k}$ generates a stochastic process with state space $\mathbb{R}^{n}$. We will say it is a solution of the impulsive differential equations with random moments of impulses (8) and it is $x(t)=a^{k} x_{0}$ for $\xi_{k}<t \leq \xi_{k+1}$.

The solution is a stochastic process.

Now, consider the expected value of the solution,

$$
E|x(t)|=\left|x_{0}\right| e^{-\lambda t} \sum_{k=0}^{\infty}|a|^{k} \frac{(\lambda t)^{k}}{k !}=\left|x_{0}\right| e^{-\lambda t(1-|a|)} .
$$

If $|a|<1$, then $E|x(t)|$ is approaching 0 (compare with the impulsive differential equation with fixed moments of impulses considered in Example 2).

We will say that conditions $(\mathrm{H})$ are satisfied if

(H1) For any initial values $\left(t_{0}, x_{0}\right): t_{0} \geq T_{0}, x_{0} \in \mathbb{R}^{n}$, the initial value problem $x^{\prime}=f(t, x(t)), x\left(t_{0}\right)=x_{0}$ has a unique solution $x(t)=x\left(t ; t_{0}, x_{0}\right)$ defined for $t \geq t_{0}$.

(H2) $f(t, 0)=0$ and $I_{k}(t, 0)=0$ for $t \geq 0, k=1,2, \ldots$.

(H3) The random variables $\left\{\tau_{k}\right\}_{1}^{\infty}$ are independent exponentially distributed random variables with a parameter $\lambda$ and $\xi_{k}=T_{0}+\sum_{i=1}^{k} \tau_{i}, k=0,1,2, \ldots$.

Definition 1 A stochastic process $y(t)$ with an uncountable state space $\mathbb{R}^{n}$ is said to be a solution of the initial value problem for the system of equations with randomly occurring impulses (2), $t_{0} \geq T_{0}$, if for any sample values $t_{k}$ of the random variables $\tau_{k}, k=1,2, \ldots$, correspondingly, the process $y(t)$ satisfies the initial value problem for the impulsive equation with fixed points of impulses (1), where the moments of impulses are defined by $T_{k}=T_{0}+\sum_{i=1}^{k} t_{i}, k=1,2, \ldots$

Remark 2 We note that if condition (H1) is satisfied, then the sample path solution of the initial value problem for the impulsive equation with impulses at random moments (2) exists for all $t>t_{0}, t_{0} \geq T_{0}$ provided that the times between two consecutive impulses $t_{k}$ are such that $\sum t_{k}=\infty$.

Remark 3 We note that if the values $t_{k}$ are values of the random variables $\tau_{k}, k=1,2, \ldots$, correspondingly, then the value $T_{k}=t_{0}+\sum_{i=1}^{k} t_{i}$ is a value of the random variable $\xi_{k}, k=$ $1,2, \ldots$

Definition 2 We will say that the stochastic processes $y(t)$ and $u(t)$ satisfy the inequality $y(t) \leq u(t)$ for $t \in J \subset \mathbb{R}$ if the state space of $v(t)=y(t)-u(t)$ is $[0, \infty)$.

Definition 3 Let $p>0$. Then the trivial solution of the impulsive differential equation with random impulses (2) is said to be $p$-moment exponentially stable if for any initial data $t_{0} \geq T_{0}$ and $x_{0} \in \mathbb{R}^{n}$, there exist constants $\alpha, \mu>0$ such that $E\left[\left\|x\left(t ; T_{0}, x_{0},\left\{\tau_{k}\right\}\right)\right\|^{p}\right]<$ $\alpha\left\|x_{0}\right\|^{p} e^{-\mu\left(t-t_{0}\right)}$ for all $t>t_{0}$, where $x\left(t ; T_{0}, x_{0},\left\{\tau_{k}\right\}\right)$ is the solution of the initial value problem for the impulsive differential equation with random impulses (2).

Remark 4 We note that the two-moment exponential stability for stochastic equations is known as exponential stability in mean square. 


\section{Main results}

In this section we will use Lyapunov functions to obtain sufficient conditions for the $p$-moment exponential stability of the trivial solution of the nonlinear impulsive random system at random moments (2).

First we obtain a formula for the solution of the following initial value problem for a scalar linear impulsive differential equation with random moments of impulses and random amplitude of jumps:

$$
\begin{aligned}
& u^{\prime}=a(t) u \quad \text { for } t \geq 0, \xi_{k}<t<\xi_{k+1}, \\
& u\left(\xi_{k}+0\right)=B_{k}\left(\tau_{k}\right) u\left(\xi_{k}-0\right) \quad \text { for } k=1,2, \ldots \\
& u\left(t_{0}\right)=u_{0},
\end{aligned}
$$

where $u, u_{0} \in \mathbb{R}, a, B_{k}: \mathbb{R}_{+} \rightarrow \mathbb{R}, k=1,2, \ldots$

\section{Lemma 3.1 Let the following conditions be fulfilled:}

1. Condition (H3) is satisfied.

2. The functions $a, B_{k} \in C\left(\mathbb{R}_{+}, \mathbb{R}\right)(k=1,2, \ldots)$.

Then the solution $u\left(t ; T_{0}, x_{0},\left\{\tau_{k}\right\}\right)$ of the initial value problem for the linear impulsive differential equation with random moments of impulses (10) is given by the formula

$$
u\left(t ; T_{0}, u_{0},\left\{\tau_{k}\right\}\right)=u_{0}\left(\prod_{i=1}^{k} B_{i}\left(\tau_{i}\right)\right) e^{\int_{t_{0}}^{t} a(s) d s} \quad \text { for } \xi_{k}<t<\xi_{k+1}, k=0,1,2, \ldots
$$

and the expected value of the solution satisfies the inequality

$$
E\left(\left|u\left(t ; T_{0}, u_{0},\left\{\tau_{k}\right\}\right)\right|\right) \leq\left|u_{0}\right| e^{\int_{t_{0}}^{t}(a(s)-\lambda) d s} \sum_{k=0}^{\infty}\left(\prod_{i=1}^{k} E\left(\left|B_{i}\left(\tau_{i}\right)\right|\right)\right) \frac{\lambda^{k}\left(t-t_{0}\right)^{k}}{k !} .
$$

Proof Choose arbitrary values $t_{k}$ of the random variables $\tau_{k}, k=1,2, \ldots$. Define the increasing sequence of points $T_{k}=\sum_{i=0}^{k} t_{i}, k=1,2,3, \ldots$, that are values of the random variables $\xi_{k}$ and consider the initial value problem for the linear impulsive differential equation with fixed points of impulses

$$
\begin{aligned}
& u^{\prime}=a(t) u \quad \text { for } t \geq 0, t \neq T_{k}, \\
& u\left(T_{k}+0\right)=B_{k}\left(T_{k}-T_{k-1}\right) u\left(T_{k}-0\right) \quad \text { for } k=1,2, \ldots \\
& u\left(t_{0}\right)=u_{0} .
\end{aligned}
$$

The solution of initial value problem (12) is given by the formula (see [4])

$$
u\left(t ; T_{0}, u_{0},\left\{t_{k}\right\}\right)=u_{0}\left(\prod_{i=1}^{k} B_{i}\left(T_{k}-T_{k-1}\right)\right) e^{\int_{t_{0}}^{t} a(s) d s} \text { for } t \in\left(T_{k}, T_{k+1}\right) .
$$

This solution generates a continuous stochastic process $u\left(t ; T_{0}, u_{0},\left\{\tau_{k}\right\}\right)$ that is defined by (11). It is a solution of the initial value problem for the linear impulsive differential equation with random moments of impulses (10). 
According to Corollary 1, formula (11), and the independence of the random variables $\tau_{k}, k=1,2, \ldots$, we obtain that the random variables $B_{k}\left(\tau_{k}\right), k=1,2, \ldots$, are independent and the expected value of the solution of the initial value problem for the scalar linear impulsive differential equation with random moments of impulses (10) satisfies

$$
\begin{aligned}
E\left(\left|u\left(t ; T_{0}, u_{0},\left\{\tau_{k}\right\}\right)\right|\right)= & \sum_{k=0}^{\infty} E\left(\left|u\left(t ; T_{0}, u_{0},\left\{\tau_{k}\right\}\right)\right| \mid S_{k}(t)\right) P\left(S_{k}(t)\right) \\
& \leq \sum_{k=0}^{\infty}\left|u_{0}\right| e^{\int_{t_{0}}^{t} a(s) d s} E\left(\left|\prod_{i=1}^{k} B_{i}\left(\tau_{i}\right)\right|\right) P\left(S_{k}(t)\right) \\
& =\left|u_{0}\right| e^{\int_{t_{0}}^{t} a(s) d s} \sum_{k=0}^{\infty}\left(\prod_{i=1}^{k} E\left(\left|B_{i}\left(\tau_{i}\right)\right|\right)\right) \frac{\lambda^{k}\left(t-t_{0}\right)^{k}}{k !} e^{-\lambda\left(t-t_{0}\right)} \\
& =\left|u_{0}\right| e^{\int_{t_{0}}^{t} a(s) d s} e^{-\lambda\left(t-t_{0}\right)} \sum_{k=0}^{\infty}\left(\prod_{i=1}^{k} E\left(\left|B_{i}\left(\tau_{i}\right)\right|\right)\right) \frac{\lambda^{k}\left(t-t_{0}\right)^{k}}{k !} .
\end{aligned}
$$

In the special case when the expected values of all amplitudes of jumps are the same, we obtain the following result.

Corollary 2 In the special case when additionally to the conditions of Lemma 3.1 the condition $E\left(\left|B_{k}\left(\tau_{k}\right)\right|\right) \leq C<\infty, k=1,2, \ldots$, holds, then the expected value of the solution $x\left(t ; T_{0}, x_{0},\left\{\tau_{k}\right\}\right)$ of initial value problem (10) satisfies the inequality

$$
E\left(\left|u\left(t ; T_{0}, u_{0},\left\{\tau_{k}\right\}\right)\right|\right) \leq\left|u_{0}\right| e^{\int_{t_{0}}^{t}(a(s)-\lambda(1-C)) d s} .
$$

In the special case when all amplitudes of jumps are deterministic, we obtain the following result.

Corollary 3 Let $B_{k}(u) \equiv b_{k}$ be nonnegative constants. Then the expected value of the solution $u\left(t ; T_{0}, u_{0},\left\{\tau_{k}\right\}\right)$ of initial value problem (10) satisfies the inequality

$$
E\left(\left|u\left(t ; T_{0}, u_{0},\left\{\tau_{k}\right\}\right)\right|\right) \leq\left|u_{0}\right| e^{\int_{t_{0}}^{t} a(s) d s} e^{-\lambda\left(t-t_{0}\right)} \sum_{k=0}^{\infty}\left(\prod_{i=1}^{k} b_{i}\right) \frac{\lambda^{k}\left(t-t_{0}\right)^{k}}{k !}
$$

Now consider the following scalar linear impulsive differential inequality with random moments of impulses:

$$
\begin{aligned}
& u^{\prime} \geq a(t) u \quad \text { for } t \geq 0, \xi_{k}<t<\xi_{k+1}, \\
& u\left(\xi_{k}+0\right) \geq B_{k}\left(\tau_{k}\right) u\left(\xi_{k}\right) \quad \text { for } k=1,2, \ldots \\
& u\left(t_{0}\right)=0
\end{aligned}
$$

where $x \in \mathbb{R}$.

Lemma 3.2 Let the following conditions be fulfilled:

1. Condition (H3) is satisfied.

2. The functions $a \in C\left(\mathbb{R}_{+},(0, \infty)\right), B_{k} \in C\left(\mathbb{R}_{+}, \mathbb{R}_{+}\right)(k=1,2, \ldots)$. 
Then the state space of the solution $u\left(t ; T_{0},\left\{\tau_{k}\right\}\right)$ of the linear impulsive differential inequalities with random moments of impulses $(16)$ is $[0, \infty)$.

Proof For any values $t_{k}$ of the random variables $\tau_{k}, k=1,2, \ldots$, we consider the increasing sequence of points $T_{k}=\sum_{i=0}^{k} t_{i}, k=1,2,3, \ldots$, and the linear impulsive differential inequalities with fixed points of impulses

$$
\begin{aligned}
& u^{\prime} \geq-a(t) u \quad \text { for } t \geq 0, t \neq T_{k}, \\
& u\left(T_{k}+0\right) \geq B_{k}\left(T_{k}-T_{k-1}\right) u\left(T_{k}\right) \quad \text { for } k=1,2, \ldots, \\
& u\left(t_{0}\right)=0 .
\end{aligned}
$$

We prove that any solution $u(t)$ of inequalities (17) is a piecewise continuous function that is nonnegative. Assume the contrary.

Suppose that there exists a point $\xi \in\left(t_{0}, T\right]$ such that $u(\xi)<0$. Then there exists a point $\xi_{1} \in\left(t_{0}, T\right]$ such that $u\left(\xi_{1}\right)<0$ and $u^{\prime}\left(\xi_{1}\right)<0$. That contradicts the first inequality of (17). Therefore $u(t) \geq 0$ on $\left[t_{0}, T_{1}\right]$.

According to the second inequality of (17), it follows that if $u\left(T_{k}\right) \geq 0$, then $u\left(T_{k}+0\right) \geq 0$. Using induction, assume that $u(t) \geq 0$ on $\left[t_{0}, T_{k}\right]$. If there exists a point $\xi_{k} \in\left(T_{k}, T_{k+1}\right]$ such that $u\left(\xi_{k}\right)<0$, then, as in the proof above, we obtain a contradiction.

Since the above proof does not depend on the points of jumps $T_{k}$, it follows that for any points $T_{k}$, the solution of (17) will be nonnegative.

Therefore the stochastic process $u\left(t ; T_{0},\left\{\tau_{k}\right\}\right)$, generated by all nonnegative functions $u(t)$, will have $[0, \infty)$ as a state space.

We will use Lyapunov functions in order to obtain sufficient conditions for the $p$ moment exponential stability of the trivial solution of systems of nonlinear impulsive differential equations with impulses occurring at random moments.

We will say that the function $V: \mathbb{R}^{n} \rightarrow[0, \infty)$ belongs to the class $\Xi$ if $V(x)$ is a continuous differentiable almost everywhere function. We will use the following known definition in the literature for the derivative of the function $V \in \Xi$ along the trajectory of a differential equation:

$$
\mathbf{D} V(t, x)=\sum_{i=1}^{n} \frac{\partial}{\partial x_{i}} V(x) f_{i}(t, x)
$$

where $t \geq 0, x \in \mathbb{R}^{n}$.

Remark 5 The function $V$ does not depend on $t$ but its derivative $\mathbf{D} V$ depends on $t$ because of the function $f$.

Theorem 3.1 Let the following conditions be fulfilled:

1. Conditions $(\mathrm{H})$ are satisfied.

2. The function $V \in \Xi$ and there exist positive constants $a, b$ such that

(i) $a\|x\|^{p} \leq V(x) \leq b\|x\|^{p}$ for $x \in \mathbb{R}^{n}$; 
(ii) for any $(t, x) \in[0, \infty) \times \mathbb{R}^{n}$, the inequality

$$
\mathbf{D} V(t, x) \leq-m(t) V(x)
$$

holds, where $m \in C\left(\mathbb{R}_{+}, \mathbb{R}_{+}\right)$and $\inf _{t \geq 0} m(t)=L \geq 0$;

(iii) there exist constants $w_{k}$ and $C$ such that $0 \leq w_{k} \leq C<1+\frac{L}{\lambda}, k=1,2, \ldots$, such that

$$
V\left(I_{k}(x)\right) \leq w_{k} V(x) \quad \text { for } x \in \mathbb{R}^{n}
$$

Then the trivial solution of the impulsive differential equations with random moments of impulses (2) is p-moment exponentially stable.

Proof Let $\left(t_{0}, x_{0}\right) \in\left[T_{0}, \infty\right) \times \mathbb{R}^{n}$ be arbitrary initial data and the stochastic process $x_{\tau}(t)=$ $x\left(t ; T_{0}, x_{0},\left\{\tau_{k}\right\}\right)$ be a solution of the initial value problem for impulsive differential equation with random impulses (2). From condition (i) it follows that $a\left\|x_{\tau}(t)\right\|^{p} \leq V\left(x_{\tau}(t)\right)$ for $t \geq T_{0}$ and

$$
E\left(\left\|x_{\tau}(t)\right\|^{p}\right) \leq \frac{1}{a} E\left(V\left(x_{\tau}(t)\right)\right), \quad t \geq T_{0} .
$$

Define the stochastic process $v_{\tau}(t)=V\left(x_{\tau}(t)\right)$ with an uncountable state space $\mathbb{R}_{+}$. From conditions (ii), (iii) we obtain that the stochastic process $v_{\tau}(t)$ satisfies the inequalities

$$
\begin{aligned}
& v_{\tau}^{\prime}(t)=\sum_{i=1}^{n} \frac{\partial}{\partial x_{i}} V\left(x_{\tau}(t)\right) f_{i}\left(t, x_{\tau}(t)\right) \leq-m(t) v_{\tau}(t) \quad \text { for } \xi_{k}<t<\xi_{k+1}, \\
& v_{\tau}\left(\xi_{k}+\right)=V\left(I_{k}\left(x_{\tau}\left(\xi_{k}\right)\right)\right) \leq w_{k} V\left(x_{\tau}\left(\xi_{k}\right)\right)=w_{k} \nu_{\tau}\left(\xi_{k}\right), \quad k=1,2, \ldots, \\
& \nu_{\tau}\left(t_{0}\right)=V\left(x_{0}\right) .
\end{aligned}
$$

Consider the initial value problem for the scalar linear differential equation with random impulses at random moments

$$
\begin{aligned}
& u^{\prime}(t)=-m(t) u(t) \quad \text { for } \xi_{k}<t \leq \xi_{k+1}, \\
& u\left(\xi_{k}+\right)=w_{k} u\left(\xi_{k}\right), \quad k=1,2, \ldots \\
& u\left(t_{0}\right)=V\left(x_{0}\right) .
\end{aligned}
$$

According to Lemma 3.1, the stochastic process

$$
u_{\tau}(t)=V\left(x_{0}\right)\left(\prod_{i=1}^{k} w_{i}\right) e^{-\int_{t_{0}}^{t} m(s) d s} \text { for } \xi_{k}<t<\xi_{k+1}, k=0,1,2, \ldots
$$

is a solution of initial value problem (22). Since $E\left(\left|w_{k}\right|\right)=w_{k} \leq C, k=1,2, \ldots$, from Corollary 2 we obtain the inequality

$$
E\left(\left|u_{\tau}(t)\right|\right) \leq V\left(x_{0}\right) e^{-\int_{t_{0}}^{t}(m(s)+\lambda(1-C)) d s} \leq V\left(x_{0}\right) e^{-(L+\lambda(1-C))\left(t-t_{0}\right)} .
$$


Consider $y(t)=u_{\tau}(t)-v_{\tau}(t), t \geq t_{0}$. The stochastic process $y(t)$ satisfies (16) with $a(t)=$ $m(t)>0$ and $B_{k}(u)=w_{k}$. According to Lemma 3.2, the state space of $w(t)$ is $[0, \infty)$, i.e., the inequality $v_{\tau}(t) \leq u_{\tau}(t), t \geq t_{0}$ holds.

From inequality (23) and condition (i) we obtain the inequalities

$$
\begin{aligned}
E\left(\left\|x_{\tau}(t)\right\|^{p}\right) & \leq \frac{1}{a} E\left(v_{\tau}(t)\right) \leq \frac{1}{a} E\left(u_{\tau}(t)\right)=\frac{1}{a} E\left(\left|u_{\tau}(t)\right|\right) \\
& \leq \frac{1}{a} V\left(x_{0}\right) e^{-(L+\lambda(1-C))\left(t-t_{0}\right)} \\
& \leq \frac{b}{a}\left\|x_{0}\right\|^{p} e^{-(L+\lambda(1-C))\left(t-t_{0}\right)}
\end{aligned}
$$

Inequality (24) proves the $p$-moment exponential stability.

Corollary 4 Let all the conditions of Theorem 3.1 be satisfied where inequality (19) is replaced by

$$
\mathbf{D} V(t, x) \leq-c\|x\|^{p}, \quad x \in \mathbb{R}^{n} .
$$

Then the inequality $E\left(\left\|x_{\tau}(t)\right\|^{p}\right) \leq \frac{b}{a}\left\|x_{0}\right\|^{p} e^{-\left((1-C) \lambda+\frac{c}{b}\right)\left(t-t_{0}\right)}$ holds.

Example 4 (Exponential stability of IDE with random moments of impulses) Let $\tau_{i}, i=$ $1,2, \ldots$, be independent exponentially distributed random variables with a parameter $\lambda$, i.e., $E\left(\tau_{i}\right)=\frac{1}{\lambda}, i=1,2, \ldots$. Consider the following initial value problem for the system of impulsive differential equations with random moments of impulses:

$$
\begin{aligned}
& x^{\prime}=-t x\left(x^{2}+y^{2}\right), \\
& y^{\prime}=t y\left(x^{2}-y^{2}\right) \quad \text { for } t \geq 0, \xi_{k}<t<\xi_{k+1}, \\
& x\left(\xi_{k}+0\right)=a x\left(\xi_{k}-0\right), \quad y\left(\xi_{k}+0\right)=b y\left(\xi_{k}-0\right) \quad \text { for } k=1,2, \ldots, \\
& x(0)=x_{0}, \quad y(0)=y_{0},
\end{aligned}
$$

where $a, b$ are constants such that $|a|<1,|b|<1$.

Consider the Lyapunov function $V(x, y)=0.5\left(x^{2}+y^{2}\right)$.

Since $0.5\left(x^{2}+y^{2}\right)=0.5\|(x, y)\|^{2}$, condition 2(i) of Theorem 3.1 is satisfied for $a=b=0.5$. In this particular case,

$$
\mathbf{D} V(t, x, y)=-t x^{2}\left(x^{2}+y^{2}\right)+t y^{2}\left(x^{2}-y^{2}\right)=-t x^{4}-t y^{4} \leq 0, \quad t \in \mathbb{R}_{+}
$$

and

$$
V(a x, b y)=a^{2} x^{2}+b^{2} y^{2} \leq C V(x, y)
$$

where $C=\max \left\{a^{2}, b^{2}\right\}$.

Therefore the conditions of Theorem 3.1 are satisfied for $m(t) \equiv 0, L=0, w_{k}=C$ and $0<C<1=1+\frac{L}{\lambda}$. 
Therefore, according to Theorem 3.1, the solution of (25) is exponentially stable in mean square, i.e.,

$$
E\left[\|(x(t), y(t))\|^{2}\right]<\alpha\left\|\left(x_{0}, y_{0}\right)\right\|^{2} e^{-\mu\left(t-t_{0}\right)}, \quad t>t_{0},
$$

where $\mu=\lambda(1-C)>0, \alpha=\frac{b}{a}=1$ and $\left\|\left(x_{0}, y_{0}\right)\right\|^{2}=x_{0}^{2}+y_{0}^{2}$.

Now, consider the system without any impulses, i.e., the system of ordinary differential equations

$$
\begin{aligned}
& x^{\prime}=-t x\left(x^{2}+y^{2}\right), \\
& y^{\prime}=\operatorname{ty}\left(x^{2}-y^{2}\right) \quad \text { for } t \geq 0 .
\end{aligned}
$$

The solution of (26) is asymptotically stable (see Figures 1 and 2) but it is not exponentially stable, since $\mathbf{D} V(t, x, y) \leq 0$ and $0.5\|(x(t), y(t))\|^{2}=V(x(t), y(t)) \leq V\left(x_{0}, y_{0}\right)=$

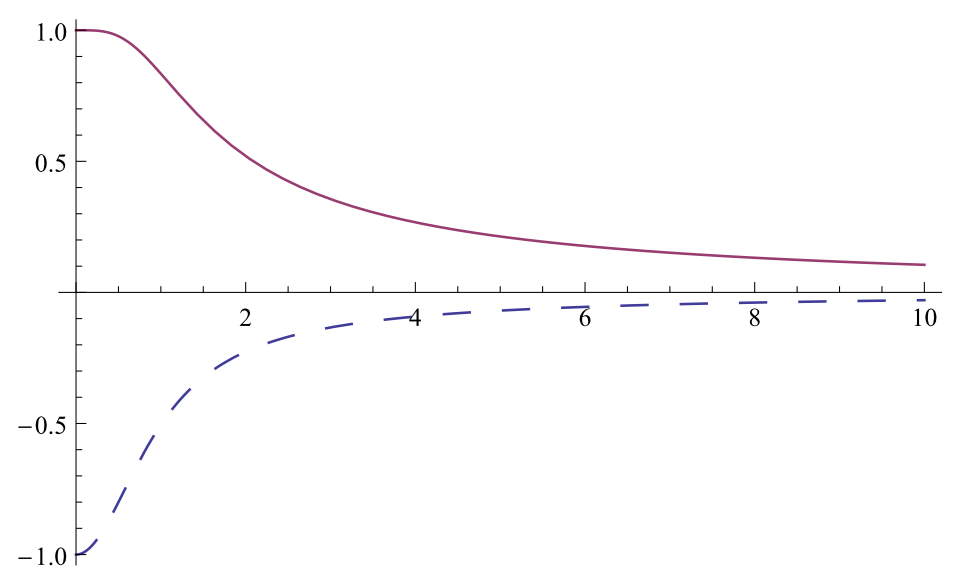

Figure 1 The graph of the solution $x(t), y(t): x(0)=-1, y(0)=1$ of $(26)$.

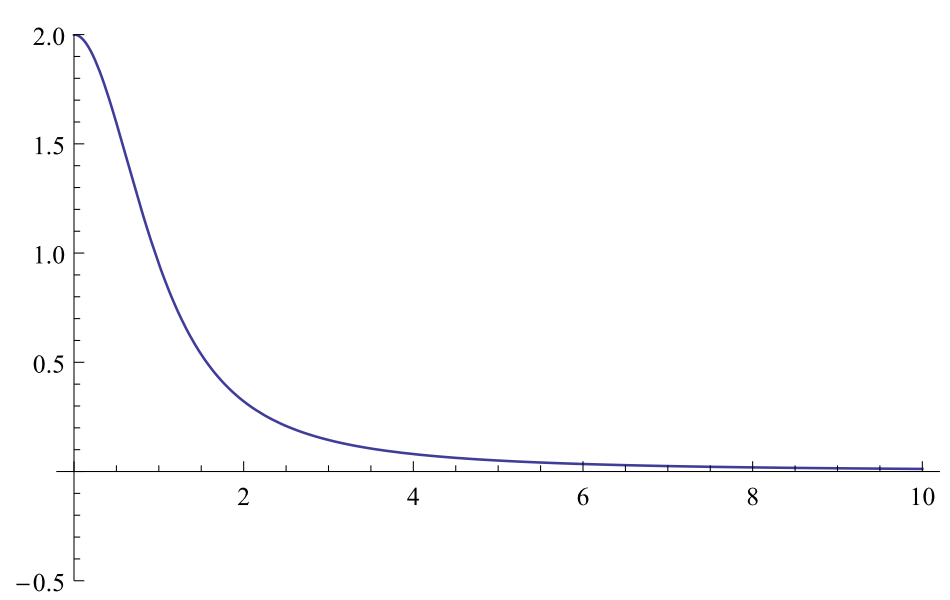

Figure 2 The graph of $x^{2}(t)+y^{2}(t)$, where $x(t), y(t): x(0)=-1, y(0)=1$ is the solution of (26). 
$0.5\left\|\left(x_{0}, y_{0}\right)\right\|^{2}$, or $\left(x^{2}(t)+y^{2}(t)\right)^{2} \leq\left(x_{0}^{2}+y_{0}^{2}\right)^{2}$ and there are no positive constants $\alpha$ and $\mu$ such that $\left(x^{2}(t)+y^{2}(t)\right)^{2} \leq \alpha\left(x_{0}^{2}+y_{0}^{2}\right)^{2} e^{-\mu t}, t>0$, for any initial points $x_{0}, y_{0}$.

Therefore, the presence of impulses at random time changes the behavior of the solution, i.e., the solution of IDE with random impulses is exponentially stable in mean square.

\section{Competing interests}

The authors did not provide this information.

\section{Authors' contributions}

The authors did not provide this information.

\section{Author details}

'Department of Mathematics, Texas A\&M University-Kingsville, Kingsville, 78363, USA. ${ }^{2}$ Plovdiv University, Tzar Asen 24 Plovdiv, 4000, Bulgaria. ${ }^{3}$ School of Mathematics, Statistics and Applied Mathematics, National University of Ireland, Galway, Ireland.

\section{Acknowledgements}

Research was partially supported by Fund Scientific Research MU13FMI002, Plovdiv University.

Received: 1 October 2013 Accepted: 21 November 2013 Published: 20 Dec 2013

References

1. Bainov, DD, Simeonov, PS: Impulsive Differential Equations: Asymptotic Properties of the Solutions. World Scientific River Edge (1995)

2. Bainov, DD, Simeonov, PS: Systems with Impulsive Effects: Stability, Theory and Applications. Ellis Horwood, Chichester (1989)

3. Hristova, S: Qualitative Investigations and Approximate Methods for Impulsive Differential Equations. Nova Science Publishers, New York (2009)

4. Lakshmikantham, V, Bainov, DD, Simeonov, PS: Theory of Impulsive Differential Equations. World Scientific, Singapore (1989)

5. Sanz-Serna, JM, Stuart, AM: Ergodicity of dissipative differential equations subject to random impulses. J. Differ. Equ. $155,262-284(1999)$

6. Wu, S, Hang, D, Meng, X: p-Moment stability of stochastic equations with jumps. Appl. Math. Comput. 152, 505-519 (2004)

7. $\mathrm{Wu}, \mathrm{H}$, Sun, J: $p$-Moment stability of stochastic differential equations with impulsive jump and Markovian switching Automatica 42, 1753-1759 (2006)

8. Naghshtabrizi, P, Hespanha, JP, Teel, AR: Exponential stability of impulsive systems with application to uncertain sampled-data systems. Syst. Control Lett. 57(5), 378-385 (2008)

9. Smalfuss, B: Attractors for nonautonomous and random dynamical systems perturbed by impulses. Discrete Contin. Dyn. Syst. 9(3), 727-744 (2003)

10. $\mathrm{Wu}, \mathrm{S}$ : The Euler scheme for random impulsive differential equations. Appl. Math. Comput. 191, 164-175 (2007)

11. Samoilenko, A, Stanzhytskyi, O: Qualitative and Asymptotic Analysis of Differential Equations with Random Perturbations. World Scientific, Singapore (2011)

12. Fu, X, Li, X: Razumikhin-type theorems on exponential stability of impulsive infinite delay differential systems. J. Comput. Appl. Math. 224(1), 1-10 (2009)

13. Liu, B, Liu, X, Teo, L, Wang, Q: Razumikhin-type theorems on exponential stability of impulsive delay systems. IMA J. Appl. Math. 71, 47-61 (2006)

14. Wang, Q, Liu, X: Exponential stability for impulsive delay differential equations by Razumikhin method. J. Math. Anal. Appl. 309(2), 462-473 (2004)

15. Wu, Q, Zhoua, J, Xiang, L: Global exponential stability of impulsive differential equations with any time delays. Appl. Math. Lett. 23(2), 143-147 (2010)

16. Xu, W, Niu, YJ, Rong, HW, Sun, ZK: p-Moment stability of stochastic impulsive differential equations and its application in impulsive control. Sci. China, Technol. Sci. 52(3), 782-786 (2009)

17. Yang, J, Zhong, S, Luo, W: Mean square stability analysis of impulsive stochastic differential equations with delays. J. Comput. Appl. Math. 216(2), 474-483 (2008)

10.1186/1687-1847-2013-372

Cite this article as: Agarwal et al.: Exponential stability for differential equations with random impulses at random times. Advances in Difference Equations 2013, 2013:372 